\title{
I. Justizpolitische Weichenstellungen 1947/48
}

Sowohl im internationalen als auch im innerzonalen Kontext legen eine Anzahl von Beobachtungen es nahe, das Jahr 1948 als Zäsur zu begreifen. Im Rahmen der internationalen Beziehungen verschärften sich 1948 mit dem Kalten Krieg auch die inneralliierten Auseinandersetzungen über Deutschland: Das Ende der Kontrollratsarbeit und die Berlin-Blockade sind in diesem Zusammenhang die herausragenden Ereignisse. Gleichzeitig fielen damals für die innere Entwicklung der SBZ politische Entscheidungen von erheblicher Bedeutung für die Folgezeit: Die Zentralisierung der Wirtschaftslenkung durch den aufgewerteten und umgebauten Apparat der „Deutschen Wirtschaftskommission“ (DWK) ist hier ebenso zu nennen wie die Stalinisierung der SED als „Partei neuen Typus“. Insgesamt verlagerten sich 1948 die politischen Machtzentren von den Ländern zur SED-Zentrale und zu den von ihr kontrollierten Apparaten, vor allem der DWK und der Deutschen Verwaltung des Innern (DVdI). Außerdem fielen in der Wirtschafts- und Finanzpolitik mit der Einführung des Zweijahresplans und der Durchführung einer eigenen Währungsreform Entscheidungen, die sich in den nachfolgenden Jahren als unumkehrbar erwiesen ${ }^{1}$.

Betroffen war auch die Justizpolitik. Bis 1947 war hier, anders als in anderen Teilen des Herrschaftssystems, die Ausbildung diktatorischer Strukturen wenig hervorgetreten. In den Jahren seit dem Zusammenbruch hatte sich die Besatzungsmacht hier, abgesehen von der rigorosen Entnazifizierung des deutschen Justizpersonals, der Einführung der Volksrichterausbildung aus eher pragmatischen Motiven und vereinzelten Eingriffen in das deutsche Normensystem, weitgehend zurückgehalten. Seit Mitte 1947 angekündigt, wurde auf diesem Feld 1948 eine programmatische Wende vollzogen, die vor allem daran erkennbar ist, daß die nun eingeleiteten Maßnahmen im Vergleich zu den vorangegangenen Jahren in ihrer Zielrichtung weniger ambivalent, sondern eindeutig auf Zentralisierung, Politisierung und Sowjetisierung ausgerichtet waren ${ }^{2}$. Die erstarkte SED ergriff nun die Initiative und verdeutlichte auch nach außen, daß sie die Zügel in der Justizpolitik in die Hand nehmen wollte. Als ihr justizpolitischer Sprecher wurde Max Fechner auserkoren, der im Oktober 1948 an die Stelle Schiffers als Präsident der DJV treten und mit der Gründung des ostdeutschen Teilstaates der erste Justizminister der DDR werden sollte.

1 Vgl. Hoffmann/Wentker, Das letzte Jahr der SBZ.

2 Eine etwas andere Gewichtung ergibt sich bei einer Betrachtung aus der Landesperspektive. So begreift Weber, Justiz und Diktatur, S. 142, 1948 als „eine Zäsur, aber keinen Wendepunkt in der Justizgeschichte Thüringens"; nach Pohl, Justiz in Brandenburg (Manuskript), S. 209, machte sich die Radikalisierung der Justizpolitik in Brandenburg „erst in der zweiten Jahreshälfte 1949“ bemerkbar. 


\section{Max Fechner}

Im Unterschied zu Schiffer war Fechner weder durch seine Ausbildung noch durch seine Tätigkeit vor 1945 für eine herausgehobene Funktion in der Justiz qualifiziert. Geboren als Sohn eines Maurers im Jahre 1892 in Rixdorf bei Berlin, erlernte er nach dem Besuch der Volksschule den Beruf eines Werkzeugmachers. Aufgewachsen im sozialdemokratischen Milieu - sein Vater war Parteimitglied -, kam er über die Sozialistische Arbeiterjugend und die Arbeitersportbewegung zur SPD, der er 1911 beitrat. Von 1914 bis 1917 diente er im Heer, wurde aber im Januar 1917 aufgrund einer Verwundung aus dem Kriegsdienst entlassen. Als Mitglied der Unabhängigen Sozialdemokratischen Partei Deutschlands, der er seit ihrer Gründung im April 1917 angehörte, wurde er nach Ausbruch der Novemberrevolution von der Belegschaft seines Betriebes in den Arbeiterrat gewählt. Nachdem er im Januar 1920 eine Demonstration gegen das Betriebsrätegesetz in seiner Firma mit organisiert hatte, wurde er fristlos entlassen, erhielt jedoch im Frühsommer 1920 beim ZK der USPD eine Anstellung als Hauptkassierer. In dieser Position hielt er dieser Partei auch dann die Treue, als sich ihr linker Flügel im Oktober 1920 mit der KPD vereinigte; im September 1922 kehrte er mit dem verbliebenen Teil der USPD wieder zur SPD zurück, deren Parteivorstand ihn als Angestellten übernahm. Auf seinen Vorschlag hin wurde dort 1924 eine kommunalpolitische Zentralstelle gegründet, die er bis 1933 leitete; gleichzeitig war er verantwortlicher Redakteur der kommunalpolitischen sozialdemokratischen Zeitschrift „Die Gemeinde“. Mit 29 Jahren wurde er Bezirksverordneter in Berlin-Neukölln und blieb bis 1929 in der Bezirksversammlung tätig; von 1924 bis 1929 war er Stadtverordneter, und von 1928 bis 1933 hatte er zudem ein Mandat im Preußischen Landtag inne. Nach eigenen Aussagen stand er als Parteifunktionär auf dem linken Flügel der SPD, da er deren Koalitionspolitik mit den bürgerlichen Parteien ablehnte. Während des Dritten Reiches blieb er seiner sozialdemokratischen Gesinnung treu und wurde im Juli 1933 von der Gestapo inhaftiert, die ihn im März 1934 wieder aus dem KZ Oranienburg entließ33. Auch danach hielt er Kontakt zu einer illegalen SPD-Gruppe um den ehemaligen Reichstagsabgeordneten Franz Künstler und wurde nach dem gescheiterten Hitler-Attentat vom 20. Juli 1944 erneut inhaftiert, kam vor der Jahreswende aber wieder freit.

Nach Kriegsende beteiligte sich Fechner an führender Stelle an der Wiederbegründung der SPD in Berlin. Er gehörte nicht nur zu den Mitunterzeichnern des Gründungsaufrufs vom 15. Juni 1945, sondern wurde neben Otto Grotewohl und Erich Gniffke zu einem der drei gleichberechtigten Vorsitzenden der Partei gewählt. Zuvor hatte er, wahrscheinlich Anfang Mai 1945, Ulbricht um ein Ge-

3 In der Untersuchungshaft beschuldigte das MfS Fechner unter Berufung auf ein Schreiben des KZ Oranienburg, bei seiner Entlassung eine „Verpflichtungserklärung" gegenüber der Gestapo unterschrieben zu haben. Fechner räumte lediglich ein, sich möglicherweise zum Stillschweigen über die Vorgänge im KZ verpflichtet zu haben: siehe Vernehmungsprotokoll Fechner, 15. 6. 1954, BStU, MfS, AU 307/55, Bd. 2, Bl. $75 \mathrm{f}$.

+ Dazu siehe u.a. Lebenslauf Fechners, 21. 6. 1964, in: SAPMO, SgY 30 1274, Bl. 3-8; Lebenslauf Fechners vom 19. 9. 1946, BAB, DP1 SE Nr. 3360, Bl. 551; Vernehmungsprotokoll Fechner o.D., BStU, MfS AU 307/55 (EV) Bd. 1, Bl. 27-34; Vernehmungsprotokoll Bruno Böttges, 1. 9. 1954, ebenda, Bl. 290; Abschrift des Lebenslaufes von Fechner, 15.1. 1951, BStU, MfS AU 307/55, Bd. 5b, Bl. 190 f.; Fricke, Justiz im Auftrag der Partei, S. 26. 
spräch über die Schaffung einer „Einheitsorganisation der deutschen Arbeiterklasse" gebeten ${ }^{5}$; als dieser nicht reagierte, orientierte sich Fechner anderweitig, so daß man diesen Schritt nicht überbewerten sollte ${ }^{6}$. Als die Kommunisten in Abänderung ihrer bisherigen Taktik ab Herbst 1945 eine Vereinigung mit den Sozialdemokraten ansteuerten, wurde Fechner freilich einer der vehementesten Einheitsbefürworter, der unter anderem Wilhelm Pieck über die SPD-Konferenz in Wennigsen bei Hannover berichtete und dabei die Chancen einer damals noch gesamtdeutsch geplanten Vereinigung als "sehr günstig“ darstellte7. Fechners Haltung läßt sich zum Teil auf die - von vielen Sozialdemokraten geteilte - Überzeugung zurückführen, daß nur eine vereinigte Arbeiterpartei den „Faschismus“ wirksam verhindern könne; zum Teil waren dabei wohl auch materielle Interessen im Spiel, denen die sowjetische Besatzungsmacht entgegenkam ${ }^{8}$.

Unmittelbar vor der Vereinigung fiel im SPD-Vorstand am 12. Februar 1946 auf Vorschlag von Gniffke die Entscheidung, daß bei der paritätischen Aufteilung der Ressorts im Zentralsekretariat Fechner gemeinsam mit Ulbricht für die Dezernate Wirtschaft, Landespolitik, Kommunalpolitik, Justiz und Allgemeines verantwortlich zeichnen sollte 9 . Im Verhältnis zwischen beiden Politikern, die erst auf dem zweiten SED-Parteitag im September 1947 zu stellvertretenden Parteivorsitzenden gewählt wurden, zeigte sich Ulbricht rasch als der überlegenere, und Fechner fiel es schwer, „überhaupt Schritt zu halten"10. Ulbricht wiederum ließ Fechner seine Überlegenheit spüren, so daß von gleichberechtigter Zusammenarbeit keine Rede sein konnte. Zudem wurde dieser von Anfang an über Entwicklungen in Wirtschaft und Polizei nicht informiert; bei der Aufteilung der Verantwortungsbereiche behielt sich Ulbricht die Leitung der Dezernate Wirtschaft und Landespolitik vor, während Fechner auf Kommunalpolitik und Justiz beschränkt wurde ${ }^{11}$. Dies bedeutete zwar, daß Fechner sich keine Kompetenzen in den Ulbricht zugesprochenen Politikfeldern anmaßte, dieser sich aber nicht scheute, auch in die Kommunal- und die Justizpolitik einzugreifen. In der Kommunalpolitik arbeitete Ulbricht auf eine Zentralisierung der Verwaltung hin; Fechner hingegen setzte auf sich selbst verwaltende Kommunen als Basis des Staats- und Gesellschaftsaufbaus ${ }^{12}$. Zwar räumte letzterer seine erstmals im Dezember 1947 angegriffene Stellung als führender Kommunalpolitiker im Zentralsekretariat nicht kampflos; bis Juli 1948 hatte er nach den Worten Erich Gniffkes jedoch „sein Mitspracherecht in allen Fragen des Verwaltungsaufbaus verlor[en]"13.

5 Fechner an Ulbricht, 28. 4. 1945, in: Keiderling, Gruppe Ulbricht, S. 287 f. Da Fechner erst am 2. Mai 1945 von der Anwesenheit Ulbrichts in Berlin erfahren hatte, ist das Datum des Briefes nicht korrekt: vgl. ebenda, S. 288, und Hurwitz, Anfänge, Teil 1, S. 84 f.

6 So Hurwitz, ebenda, S. $87 \mathrm{f}$.

7 Vgl. Bouvier/Schulz, SPD, S. 75; Notizen Piecks über ein Gespräch mit Fechner, 15. 10. 1945, in: Dokumente zur Geschichte der kommunistischen Bewegung, Bd. 2, S. $598 \mathrm{f}$. (dort auch das Zitat).

\& Vgl. Gniffke, Jahre, S. 109; Bouvier/Schulz, SPD, S. 81.

9 Gniffke, Jahre, S. 148; Kaiser, Zentrale der Diktatur, S. 62; Amos, Justizverwaltung, S. 76.

10 Bemerkungen Scheeles zur Arbeit im ZS kurz nach der Vereinigung, BStU, MfS AU 307/55, Bd. 6, Bl. 214.

11 Abschrift des Lebenslaufes von Fechner, 15. 1. 1951, BStU, MfS AU 307/55, Bd. 5b, Bl. 191; Erinnerungen des Genossen Max Fechner, 30. 1. 1965, SAPMO, SgY 30 1274, Bl. 53 f. Zum Verhältnis Fechner-Ulbricht vgl. Gniffke, Jahre, S. $181 \mathrm{f}$.

12 Ebenda, S. 294; Stern, Ulbricht, S. 142.

13 Gniffke, Jahre, S. 294-296, 305 f., 327 (dort auch das Zitat). 
Somit blieb für den Parteifunktionär Fechner mit dem Justizressort ein von der SED als nachgeordnet betrachteter Aufgabenbereich, für den er aufgrund seiner Erfahrung und Ausbildung am wenigsten qualifiziert war. Er wurde daher weitgehend von den Vorlagen der Justizabteilung beim Zentralsekretariat und damit von Karl Polak, dem ,juristische[n] Mentor Ulbrichts“ 14 abhängig. In dieser Situation erhielt Fechners persönlicher Referent, Günter Scheele, mit dem er seit 1928 eng verbunden war, für ihn eine besondere Bedeutung als Vertrauensperson und fachlicher Zuarbeiter. Geboren 1905, hatte Scheele im Anschluß an eine Lehrerausbildung das Abitur nachgeholt und parallel zu seiner Lehrtätigkeit ein Studium der Philosophie, Psychologie, Geschichte und Soziologie in Berlin absolviert, das er 1932 mit der Promotion abschloß15. Unmittelbar nach seinem SPD-Beitritt im Jahre 1928 kam er mit Fechner zusammen und unterstützte diesen besonders bei den Wahlen von 1930 und 193316. Im Dritten Reich blieb er bis 1937 in lockerem Kontakt mit Fechner und Franz Künstler17. 1937 trat er in die NSDAP ein ${ }^{18}$. Als auf Beschluß des SPD-Zentralausschusses Fechner nach dem Krieg einen persönlichen Referenten zugesprochen bekam und Scheele auf seinen Wunsch diese Funktion übernahm, verständigten sich beide darauf, daß man Scheeles NSDAPMitgliedschaft zwar zugeben, gleichzeitig aber verbreiten solle, er sei angeblich auf Veranlassung von Fechner und Künstler in die Partei eingetreten, um für seine Auftraggeber bedeutsame Interna in Erfahrung zu bringen ${ }^{19}$. Diese Taktik ging auf, und Scheele wurde, nachdem er im Januar 1946 auf Betreiben Fechners vom geschäftsführenden Vorstand des SPD-Zentralausschusses rehabilitiert worden war, zunächst dessen Mitarbeiter im SPD-Vorstand und wechselte dann als dessen persönlicher Sekretär ins SED-Zentralsekretariat ${ }^{20}$. Damit gewann Fechner, wie Gniffke treffend bemerkte, „einen hochintelligenten Sekretär, der ihm nicht nur die schriftlichen Arbeiten abnahm und ihm die Vorträge ausarbeitete, sondern ihm auch sein Buch ,Wie konnte es geschehen?'21 schrieb"22. Scheele hatte Fechner zudem durch zwei Broschüren, in denen mit den Vereinigungsgegnern und Kurt

14 So die zutreffende Bezeichnung von Markovits, Abwicklung, S. 155.

$15 \mathrm{Zu}$ Scheele vgl. dessen Erinnerungen, SAPMO, SgY 30 1721; Lebenslauf Scheeles, 24.4. 1951, SAPMO, DY 30 IV 2/11/177, Bl. 61-65, hier 61.

16 Ebenda, Bl. 62.

17 Vernehmungsprotokoll Fechner, 24. 7. 1953, BStU, MfS AU 307/55, Bd. 1, Bl. 51.

18 Laut NSDAP-Mitgliedskartei, BAB, wurde er am 1.5. 1937 aufgenommen und erhielt die Mitgliedsnummer 4827162; vgl. Amos, Justizverwaltung, S. $111 \mathrm{f}$.

19 Vernehmungsprotokoll Fechner, 24. 7. 1953, BStU, MfS AU 307/55, Bd. 1, Bl. 51 f.; Ergänzungen Scheeles zu seinem Bericht vom 20.7. 1953 mit dem Titel: „Zu meiner Lüge vom illegalen Auftrag“, ebenda, Bd. 6, Bl. 218-220.

20 Vermerk betr. Verhalten Scheeles 1933-1945, 8. 8. 1946, gez. Gniffke, BStU, MfS AU Nr. 306/55, Bl. 16f. Vgl. auch die Erklärung im "Neuen Deutschland“" vom 24. 8. 1946; damit reagierte das SED-Zentralsekretariat auf ein SPD-Flugblatt, in dem das Gerücht verbreitet wurde, daß Fechners Privatsekretär NSDAP-Mitglied gewesen sei; vgl. BAB, DP1 VA Nr. 1009, Bl. 27, und Protokoll der Zentralsekretariatssitzung, 17. 8. 1946, SAPMO, DY 30 IV 2/2.1/24, TOP 13.

21 Der genaue Titel lautet: Wie konnte es geschehen? Auszüge aus den Tagebüchern und Bekenntnissen eines Kriegsverbrechers, 6. Aufl., Berlin o.J. [1945/46]. Der „Kriegsverbrecher“ war Joseph Goebbels, dessen Aussagen lediglich nachempfunden wurden: vgl. ebenda, S. 131. Tjulpanow ermunterte Fechner, sein Buch drucken zu lassen, und sicherte ihm Papier für eine Millionenauflage zu: vgl. Gniffke, Jahre, S. 109, und Anordnung Nr. 10 vom 1. 3. 1946 der SMAD-Propagandaabteilung, BStU, MfS AU 307/55, Bd. 5b, Bl. 178. Vgl. insgesamt dazu Suckut, Als wir in den Hof, S. 176 .

22 Gniffke, Jahre, S. 69 f. 
Schumacher abgerechnet wurde, "gleichsam nach oben geschrieben "23. Obgleich kein Jurist, arbeitete Scheele Fechner auch dessen justizpolitische Vorträge und Beiträge aus, in denen die „Demokratisierung der Justiz“ im Sinne der SED gefordert wurde ${ }^{24}$.

War Fechner somit als justizpolitischer Sprecher seiner Partei in mehrfacher Hinsicht eingeengt, kontrolliert und lenkbar - Ulbricht verzichtete nie auf seinen Anspruch, auch persönlich in Justizangelegenheiten einzugreifen -, so bedeutete für ihn die Ernennung zum Chef der DJV seine endgültige Verdrängung aus Macht und Einfluß. Für die SED-Führung stand spätestens mit ihrem Antrag vom Juli 1948 beim Obersten Chef der SMAD, Marschall Wassili Sokolowski25, fest, daß Fechner Schiffers Nachfolger werden sollte. Es kostete indes einige Mühe, Fechner zur Übernahme des Präsidentenamtes zu bewegen. Scheele gegenüber sprach er in diesem Zusammenhang von einer "der schwersten Entscheidungen in seinem Leben“. „Er empfand diesen Beschluß“, so berichtete Scheele, „als ein Abschieben aus der Politik. Er sagte den beiden Vorsitzenden diese seine Meinung, sie könnten es leichter haben, er könnte gehen und in Schöneiche Bürgermeister machen. Gen[osse] W. Pieck erklärte ihm, daß es jetzt notwendig wird, daß leitende Funktionäre aus dem Apparat in die Verwaltung müßten, es wäre kein Abschieben. Sein Einwand, daß er kein Jurist sei, wurde mit dem Hinweis auch von seiten der Freunde dadurch entkräftet, daß gute Juristen in der Justizverwaltung seien, es käme auf eine gute politische Führung an." Dem Druck Piecks und der SMAD hielt Fechner nicht stand, machte nunmehr aber die Übernahme des Präsidentenamtes davon abhängig, daß Scheele als sein persönlicher Mitarbeiter ebenfalls in die DJV überwechselte ${ }^{26}$. Nachdem auch Pieck dies befürwortet hatte, faßte das Zentralsekretariat am 13. September 1948 einen entsprechenden Beschluß. Bereits eine Woche zuvor war entschieden worden, Fechner für das Präsidentenamt vorzuschlagen; gleichzeitig hatte das Zentralsekretariat - wohl um diesem nicht das Gefühl zu geben, , abgeschoben' zu werden - bekräftigt, „daß durch die Übernahme des Postens seine Funktionen im Zentralsekretariat nicht berührt werden"27. Mit SMAD-Befehl Nr. 158 wurde er schließlich am 2. Oktober zum DJV-Präsidenten ernannt ${ }^{28}$. Formell blieb Fechner zwar Mitglied des Zentralsekretariats; dem im Januar 1949 gebildeten Politbüro gehörte er freilich nicht mehr an. Damit war er endgültig entmachtet und auf ein Arbeitsgebiet festgelegt worden, auf dem er sich aufgrund seiner mangelnden Qualifikationen am wenigsten profilieren und am besten gelenkt werden konnte.

23 So Suckut, Als wir in den Hof, S. 177.

24 Bericht Scheeles, BStU, MfS AU 307/55, Bd. 6, Bl. 170.

25 Siehe Badstübner/Loth, Pieck-Aufzeichnungen, S. 235. In der SMAD galt Fechner als „amorphe, nicht so militante Figur“, wurde aber geschätzt, da er „viele sehr gute Dokumente zur Entlarvung Schumachers geliefert hat". Zit. nach Bordjugow, SMAD und SED, S. 288.

26 Bericht Scheeles, BStU, MfS AU 307/55, Bd.6, Bl. $171 \mathrm{f}$. Gegenüber dem MfS gab Fechner am 25. 7. 1953 zu: „Ich muß sagen, daß ich ohne Scheele meine Funktionen nicht hätte durchführen können.“ BStU, MfS, AU 307/55, Bd. 1, Bl. 53; vgl. Suckut, Als wir in den Hof, S. 177.

27 Protokolle der Zentralsekretariatssitzungen vom 6.9. und 13.9. 1948, SAPMO, DY 30 IV 2/2.1/ 228 und 229, TOP 18 und 8.

28 Vgl. Inventar, S. 160. 


\section{Die justizpolitische Offensive der SED}

Nach ersten Ankündigungen in der zweiten Jahreshälfte 1947 setzte im Januar 1948 eine justizpolitische Offensive der SED ein, die, unterstützt von der SMAD, das Rechtsleben in der SBZ und der späteren DDR nachhaltig verändern sollte ${ }^{29}$. Das erste Signal in diese Richtung setzte Fechner auf einer Justizkonferenz in Schwerin am 7. Juni 1947. Sein Vortrag unterschied sich von den bisherigen Äußerungen aus der SED vor allem durch das Eingeständnis, bisher die Justiz vernachlässigt zu haben, die im Vergleich zu dem „Erfolg des demokratischen Aufbaues auf den anderen Gebieten des gesellschaftlichen Lebens" zurückgeblieben sei. Und er fuhr fort: „Das Zentralsekretariat hat der Justiz große Aufmerksamkeit zugewandt, und ich darf versichern, daß nunmehr nach der ersten Phase unseres demokratischen Aufbaues die Justiz stärker als bisher in den Mittelpunkt unserer Arbeit gestellt werden wird." Als die beiden dringendsten Zukunftsaufgaben nannte er den „Ausbau und die erfolgreiche Ausgestaltung der Volksrichterschulen" sowie die Koordinierung der laufenden Gesetzgebung der Länderparlamente ${ }^{30}$.

Die Planungen zur Verwirklichung des zweiten Vorhabens waren bereits angelaufen. Denn am 23. Mai war nach einer Besprechung Fechners mit Melsheimer, Schäfermeyer und Polak festgelegt worden, zu diesem Thema am 21./22. Juni eine Sitzung des SEDAusschusses für Rechtsfragen abzuhalten, an der je ein SED-Vertreter der Rechtsausschüsse der Landtage und je ein SED-Mitarbeiter aus den Landesjustizministerien teilnehmen sollten ${ }^{31}$. Diese Zusammenkunft ist nicht nur deshalb von Bedeutung, weil auf ihr zentrale laufende Gesetzgebungsvorhaben wie etwa die Kassationsgesetze, die Gesetze zur Einrichtung der Verwaltungsgerichte oder die Wirtschaftsstrafverordnung - vorangebracht wurden, sondern auch weil Fechner hier erstmals verkündete, daß das SED-Zentralsekretariat sich in der SBZ als „politische Zentralinstanz" betrachte, die in Ermangelung einer staatlichen Zentrale die Aufgabe habe, durch Koordinierung der Gesetzesprojekte für „eine einheitliche politische Linie“ zu sorgen. Streitig gemacht wurde Fechner dieser Anspruch von Melsheimer, der forderte, „daß die ZV [Zentralverwaltung] für Justiz in die Lage versetzt wird, die Zoneneinheit zu garantieren“. Fechner machte demgegenüber jedoch unmißverständlich klar: „Über der ZV steht als höchste politische Instanz das ZS. “ Mit dieser Auffassung setzte er sich auf der Konferenz durch, die auf Antrag Nathans beschloß, das Zentralsekretariat möge "die Fraktion in den Landtagen [...] verpflichten, das ZS in Kenntnis zu setzen und ihm Gelegenheit zur Stellungnahme zu geben: 1. bevor von der betreffenden Fraktion ein Gesetz durch Initiativantrag eingebracht wird, 2. bevor von der Fraktion an anderen Vorlagen Änderungen vorgenommen werden" ${ }^{32}$.

29 Amos, Justizverwaltung, S. 58, verlegt die „parteipolitische Offensive der SED im Rechtsleben der Sowjetischen Besatzungszone “ ins Frühjahr 1947, übersieht dabei aber, daß die von ihr geschilderten Maßnahmen - abgesehen von den Beschlüssen zur Volksrichterausbildung - alle dem Aufbau und der Konsolidierung des SED-Justizapparats dienten.

30 Referat auf der Justizkonferenz am 7.6. 1947 in Schwerin, BAB, DP1 VA Nr. 6992.

31 Abteilung Justiz an Ulbricht/Fechner, 23. 5. 1947, BAB, DP1 VA Nr. 6596, B1. 153.

32 Stenographische Niederschrift über die Sitzung des Ausschusses für Rechtsfragen am 21. und 22. 6. 1947, SAPMO, DY 30 IV 2/1.01/51, Bl. 4 f., 9 f., 13, $130 \mathrm{f} ., 134$. 
Auf dem zweiten SED-Parteitag (20.-24. September 1947) widmete Ulbricht im Rahmen seines ausführlichen Referates "Der demokratische Neuaufbau in Wirtschaft und Verwaltung" zwar auch der Justizreform einige Bemerkungen, beschränkte sich dabei jedoch im wesentlichen auf eine Bilanz des bisher Erreichten und einige bereits mehrfach zuvor von der SED geäußerte Allgemeinplätze: Die Justiz solle „unabhängig sein, jedoch nur im Rahmen der demokratischen Gesetzgebung "; den Absolventen der Volksrichterlehrgänge sei „der Aufstieg in die höchsten Justizfunktionen“ zu gewährleisten; der "Grundsatz der Unabsetzbarkeit der Richter“ könne nicht erneuert werden. Auch seine „Erwartung [...], daß die Justiz demokratisch und volksverbunden wird, daß sie die Bestimmungen unserer Verfassungen im demokratischen Geiste anwendet und an der Entwicklung eines fortschrittlichen demokratischen Rechtes mitarbeitet", ging nicht über das hinaus, was bereits seit längerem von den SED-Juristen gefordert wurde ${ }^{33}$. Der zweite Parteitag im allgemeinen und die justizpolitischen Äußerungen Ulbrichts im besonderen können somit keineswegs als „das entscheidende Signal“ für einen unmittelbar bevorstehenden „schonungslosen Ausbau des Parteieinflusses auf die Justiz" gelten ${ }^{34}$.

Die SED beschränkte sich vielmehr im Herbst 1947 auf eine weitere Effektivierung ihres Justizapparats ${ }^{35}$ und auf parteiinterne Vorschläge. Polak und Schäfermeyer etwa kritisierten im November in einer Vorlage für das Zentralsekretariat, daß die DJV ,in ihrem derzeitigen Bestande nicht in der Lage ist, den anwachsenden Aufgaben, die die Demokratisierung der Justiz erfordert, gerecht zu werden“. Dies führten sie ausschließlich auf das Vorherrschen bürgerlicher Politiker an der Spitze der DJV und der Landesjustizministerien sowie auf die weiterhin bestehende Dominanz von "Juristen alter bürgerlicher Schule" in der Justiz insgesamt zurück. „Es ergibt sich das Bild“, so formulierten sie in Anlehnung an Fechner, „daß die Justiz der allgemeinen Entwicklung hinterherhinkt.“ Daher müsse die DJV die Schulung des gesamten Justizpersonals systematisieren und politisieren sowie die Kontrolle der Rechtsprechung verstärken, um gegebenenfalls „regulierend eingreifen zu können“. Für diese Aufgaben müßten neben Melsheimer zwei weitere Vizepräsidenten von der SED eingestellt werden: der damalige 2. Berliner Bürgermeister Heinrich Acker und der Leipziger Oberbürgermeister Erich Zeigner, "der zugleich die geeignete Person im Falle des Ablebens von Schiffer wäre"36. Sehr weit kamen sie mit diesem Vorschlag jedoch nicht: Das Zentralsekretariat sah am 1. Dezember nur noch einen weiteren Vizepräsidenten für die DJV vor - Staatssekretär Fritz Geyer aus Dresden ${ }^{37}$. Aber auch dieser blieb in Sachsen, so daß sich am Parteieinfluß auf die DJV vorerst nichts änderte.

Der innerparteiliche Diskussionsprozeß wurde erst mit der dritten Tagung des SED-Ausschusses für Rechtsfragen am 3./4. Januar $1948 \mathrm{zu}$ einem vorläufigen

33 Protokoll des 2. SED-Parteitages, S. 340-342.

34 So Heil, Verwaltungsgerichtsbarkeit, S. 178.

35 Siehe Arbeitsplan für die Abt. Justiz, Oktober-Dezember 1947, BAB, DP1 VA Nr. 6596, Bl. 126137.

36 Abteilung Justiz an Ulbricht/Fechner, 8. 11. 1947, sowie der beigefügte Entwurf einer Vorlage betr.: Zentralverwaltung für Justiz, ebenda, Bl. 113-115.

37 Protokoll der Zentralsekretariatssitzung vom 1. 12. 1947, SAPMO, DY 30 IV 2/2.1/151. 
Abschluß gebracht. Seit Oktober 1947 von der Justizabteilung geplant ${ }^{38}$ und im Dezember vom Zentralsekretariat gebilligt ${ }^{39}$, diente diese Konferenz der Vorbereitung einer umfassenden Behandlung der Justizfragen auf der Parteivorstandssitzung am 14./15. Januar. Bereits die Tagesordnung zeigt, daß die SED ihren Einfluß nicht nur allgemein im Justizwesen, sondern vor allem in zwei stark politisierten Aufgabenfeldern der Justiz, der Aburteilung von NS-Verbrechen nach Befehl Nr. 201 und der Einführung eines neuen Wirtschaftsstrafrechts, verstärken wollte. Voraussetzung für die "weitere Demokratisierung der Justiz“ war, wie Fechner darlegte, ein verstärktes Engagement der Partei, die „der Motor zur demokratischen Umgestaltung" werden solle. Seine allgemein gehaltene Einleitung wurde durch eher theoretische Ausführungen Schäfermeyers und praktische Darlegungen Hilde Neumanns zur Intensivierung der Parteiarbeit bei der Gesetzgebung, der Rechtsprechung, der Personalpolitik und der Schöffenschulung ergänzt. Am deutlichsten wurde Ulbricht, der insbesondere vier unmittelbar bevorstehende Aufgaben konkret benannte: erstens, die "reaktionären Kräfte" mit Hilfe von Befehl Nr. $204^{40}$ „aus der Justiz hinauswerfen“, zweitens, den im Gesetzgebungsverfahren „bei uns eingerissen[en]“ Föderalismus beseitigen, drittens, die Justizverwaltung zentralisieren, was jedoch, viertens, voraussetze, daß in der DJV „Ordnung" geschaffen werde durch die Verstärkung der kommunistischen und die gleichzeitige Schwächung der bürgerlichen Kräfte. Hauptträger dieser Aktionen sollte Ulbricht zufolge vor allem die Parteiorganisation sein. Ausdrücklich begrüßt wurden Ulbrichts und Fechners Beiträge von Melsheimer, der daraus zu Recht entnahm, „daß die Partei und das Zentralsekretariat in den Fragen der Justiz von jetzt $\mathrm{ab}$ einen neuen Kurs gehen ". Er betonte seinerseits den engen $\mathrm{Zu}$ sammenhang der geplanten staatlichen und gesellschaftlichen Umgestaltung mit der neuen Justizpolitik: „Man soll beherzigen, daß es ein alter revolutionärer und demokratischer Grundsatz ist, daß man einen Staat dann umwandelt, wenn man zwei Dinge in der Hand hat: die Polizei und die Justiz. Die Polizei hat man in der Hand, die Justiz noch nicht. Daß wir sie in die Hand bekommen, sollte unser Ziel sein!" 41

In Vorbereitung der Parteivorstandssitzung verabschiedete das Zentralsekretariat als die maßgebliche Zentralinstanz der SED am 8. Januar eine Reihe weitreichender Beschlüsse zur Umsetzung der Vorgaben des Ausschusses. Erstens wollte die SED personalpolitisch in die Offensive gehen: 50 geeignete Genossen sollten als Richter oder Staatsanwälte bestimmt, weitere 50 bereits in der Justiz tätige SED-Mitglieder auf einem Speziallehrgang der Deutschen Verwaltungsakademie für leitende Stellen in der Justiz vorbereitet und als weitere DJV-Vizepräsidenten Geyer und Benjamin vorgeschlagen werden. Zweitens billigte das Zentralsekreta-

38 Siehe Arbeitsplan für die Abt. Justiz, Oktober-Dezember 1947, BAB, DP1 VA Nr. 6596, Bl. 126.

39 Siehe die Protokolle der Zentralsekretariatssitzungen vom 4. und 22.12. 1947, SAPMO, DY 30 IV, $2 / 2.1 / 153,159$.

40 SMAD-Befehl Nr. 204 vom 23. 8. 1947 schloß ehemalige Mitglieder der NSDAP oder ihrer Gliederungen vom Richter- und Staatsanwaltschaftsberuf aus: siehe dazu Kap. B.IV.4.

41 Stenographische Niederschrift über die 3. Tagung des Ausschusses für Rechtsfragen beim Zentralsekretariat der SED, 3./4 1. 1948, SAPMO, DY 30 IV 2/1.01/70, Bl. 5-12, 152-158, 158-168, 197206, 231-233. Das Zitat aus Melsheimers Beitrag (Bl. 233) auch in: Im Namen des Volkes? Katalog, S. 15. 
riat einen Plan zur Justizreform: Darin ging es vornehmlich um die Entlastung der Justiz - etwa durch die Übertragung von Vormundschafts- und Grundbuchangelegenheiten auf die Verwaltung und Maßnahmen zur Verkürzung und Vereinfachung von Strafprozessen -, aber auch um eine Stärkung der Staatsanwaltschaft gegenüber anderen staatlichen Institutionen und eine Stärkung des Gerichts gegenüber der Verteidigung und dem Angeklagten im Strafprozeß. Drittens stimmte das Zentralsekretariat einer Stellungnahme des Rechtsausschusses zur Durchführung von Befehl Nr. $201 \mathrm{zu}$, und viertens wurden zwei den innerparteilichen Justizapparat betreffende Maßnahmen ins Auge gefaßt: die Bildung von Arbeitsgemeinschaften „aller mit Justizarbeit betrauten Genossen“ und die Herausgabe eines juristischen Informationsblattes für SED-Juristen. Das wichtigste Ergebnis stellte jedoch die Billigung der justizpolitischen Resolution des Rechtsausschusses dar, die „der innerorganisatorischen Verwendung“ dienen sollte. Damit wurde die Forderung, „daß sich unsere Partei mit den Justizproblemen ebenso intensiv beschäftigt wie mit allen anderen politisch wichtigen Fragen", zur offiziellen Leitlinie der Partei. Daraus ergaben sich als Hauptaufgaben für die SED-Gremien, die Justiz stärker in der Parteiarbeit zu thematisieren, und für die SED-Juristen, ,in laufender Schulungsarbeit die akuten Probleme unseres demokratischen Aufbaus durch[zu]arbeiten“ sowie eine Optimierung der Personalpolitik, vor allem bei der Kandidatenauswahl für die Volksrichterlehrgänge ${ }^{42}$.

Vor dem Parteivorstand, der sich am 14./15. Januar 1948 zum ersten Mal mit Fragen der Justiz befaßte, hielt Fechner erneut sein Referat über die „Demokratisierung der Justiz", das mit seinen Ausführungen vom 3./4. Januar weitgehend übereinstimmte ${ }^{43}$. Wie es den bereits damals üblichen Gepflogenheiten entsprach ${ }^{44}$, bestätigte der Parteivorstand die Resolution des Zentralsekretariats ohne Gegenstimmen ${ }^{45}$, so daß nunmehr die justizpolitische Leitlinie der Partei auch offiziell festlag. Zwar wollte die SED-Führung die Resolution nicht veröffentlichen; im Anschluß an einen Antrag, Fechners Referat drucken zu lassen, erteilte das Zentralsekretariat in der Sitzung vom 20. Januar diesem aber den Auftrag, „alle bedeutsamen Publikationen der Mitarbeiter der Justizabteilung über die Frage ,Demokratisierung der Justiz' zu einem Handbuch zusammenzustellen“" 46 . Mit dem noch im selben Jahr erschienenen „Handbuch" ${ }^{47}$, das neben einem einleitenden Artikel Fechners („Volk und Justiz“) nur Beiträge von SED-Juristen enthielt, machte die SED somit auch öffentlich ihren Führungsanspruch bei der Transfor-

42 Protokoll der Zentralsekretariatssitzung, 8. 1. 1948 mit Anlagen 1-3, SAPMO, DY 30 IV 2/2.1/ 162. Die Resolution des rechtspolitischen Beirats in: Amos, Justizverwaltung, S. 231-233.

43 Stenographisches Protokoll der Parteivorstandstagung am 14./15. 1. 1948, SAPMO, DY 30 IV 2/ 1/38, Bl. 104-125. Die Rede enthielt unter anderem die programmatische Aussage: „Wir haben bisher die Justiz links oder besser gesagt rechts liegengelassen. Das können wir uns jetzt aber nicht mehr erlauben. Die Partei muß ihre ganze Kraft jetzt mit dafür einsetzen, daß die undemokratische und volksfremde Formaljustiz endgültig liquidiert wird und die fortschrittlichen Kräfte offensiv werden gegen die Bestrebungen, das alte Recht wieder herzustellen." (Bl. 106).

44 Vgl. Friedrich u. a., Entscheidungen der SED, S. $18 \mathrm{f}$.

45 Stenographisches Protokoll der Parteivorstandstagung, 14./15. 1. 1948, SAPMO, DY 30 IV 2/1/ $38, \mathrm{Bl} .144$.

46 Ebenda und Protokoll der Zentralsekretariatssitzung, 20. 1. 1948, SAPMO, DY 30 IV 2/2.1/167, TOP 10 (dort das Zitat).

47 Fechner, Beiträge. 
mation des Justizwesens deutlich. Es ist sicher kein Zufall, daß die Entscheidung der SED, der Justiz nunmehr ihre eigene Prägung aufzudrücken und sich verstärkt im Justizwesen zu engagieren, im unmittelbaren Vorfeld ihrer Umwandlung zu einer „Partei neuen Typus“ fiel. Denn damit verbunden war der Führungsanspruch der SED auf allen Gebieten des öffentlichen Lebens sowie die Verpflichtung aller Parteileitungen, sich die entsprechende Kompetenz anzueignen ${ }^{48}$.

\section{Die Justizpolitik der SMAD 1947/48}

Im Unterschied zu den Jahren 1945/46 trat Ende 1947/Anfang 1948 die SMADRechtsabteilung hinter der SED-Führung als maßgeblicher justizpolitischer Akteur zurück. Denn sie gestand bei wichtigen Gesetzgebungsvorhaben wie dem Kassationsgesetz und der Einführung der Verwaltungsgerichtsbarkeit dem SEDZentralsekretariat eine Koordinierungsfunktion zu und verzichtete weitgehend auf direkte Einflußnahmen auf zentraler Ebene ${ }^{49}$. Dabei war die SMAD - wie der Erlaß von Befehl Nr. 201 am 16. August 1947 zeigt - damals an Justizfragen weder uninteressiert noch inaktiv. Mit dessen Durchführung wurde freilich nicht nur die DJV, sondern auch die DVdI beauftragt, und die Polizeiorgane gewannen dabei maßgebliche Kompetenzen ${ }^{50}$. Es ist daher zu vermuten, daß auch innerhalb der SMAD die Innenverwaltung - und nicht die Rechtsabteilung - für die Ausgestaltung des Befehls verantwortlich zeichnete. Zudem wurde von einem namentlich nicht bekannten SMAD-Offizier Ende August/Anfang September 1947 im Rahmen einer allgemeinen Kritik am Stand der „Demokratisierung“ in der SBZ auch den Gerichten „eine versteckte Sabotage“ vorgeworfen und Innenverwaltung und SMAD-Rechtsabteilung dazu aufgefordert, „die richtige Durchführung des Befehls Nr. 201 [zu] sichern und auch [zu] berücksichtigen, daß wir in der Justiz offensichtliche Unordnung haben, daß in der Landesjustizverwaltung und in den örtlichen Organen sehr viele reaktionäre Elemente sitzen" 51 . Auch Major Pjotr Semjonowitsch Ljulka von der SMAD-Informationsverwaltung bekundete gegenüber Schäfermeyer am 28. November 1947 die Unzufriedenheit der Abteilung „über die allgemeine Lage in der Justiz, insbesondere die Kaderfrage“ und bezeichnete - ähnlich wie die SED - die Justiz „als ein vernachlässigtes Gebiet“. „Es sei“, so fuhr er fort, „das richtige Bewußtsein über die Bedeutung der Justiz und ihre Behandlung als zentrale Frage des künftigen Staatsaufbaus noch nicht vorhanden, was insbesondere dadurch zum Ausdruck käme, daß die Kaderfrage nicht zentral genug gestellt würde." Ljulka verband diese heftigen Vorwürfe mit einer deutlichen Kritik an der SMAD-Rechtsabteilung, die "noch zu schwach besetzt

48 So Franz Dahlem auf der Parteivorstandstagung am 12./13. 5. 1948, in: Friedrich u.a., Entscheidungen der SED, S. 81; zum Gesamtprozeß der Umwandlung der SED siehe Malycha, Partei von Stalins Gnaden.

49 Siehe dazu Kap. A.V.2 und 3.

so Siehe dazu Kap. B.VI.

51 Undatiertes Redemanuskript (Ende August/Anfang September 1947), in: Badstübner/Loth, Pieck-Aufzeichnungen, S. 161-172, hier 170. Auch der MGB-Bevollmächtigte Kowaltschuk kritisierte im Juni 1948 die deutschen Justizorgane, die die „demokratischen Umwälzungen“ nicht unterstützten: vgl. Petrov, Apparate des NKVD/MVD, S. 153. 
sei und sich zu sehr in Einzelfragen verliere" ${ }^{52}$. Auch die Kaderabteilung des ZK der KPdSU stimmte in diese Klagen ein, als sie 1947 Karassjow vorwarf, die Justizreform nicht ernsthaft zu betreiben und das Justizwesen nicht entschlossen genug von Nazis und Reaktionären zu säubern ${ }^{53}$. Die innersowjetische Kritik an Karassjow verstärkte sich $1948^{54}$. All dies deutet darauf hin, daß auch in der Militärverwaltung seit dem zweiten Halbjahr 1947 ein Umdenken im Hinblick auf das Justizwesen in der SBZ eingesetzt hatte. Gleichzeitig stand die SMAD-Rechtsabteilung unter Rechtfertigungszwang nach innen und trat zu Beginn des Jahres 1948 kaum nach außen auf. Bei der Tagung des SED-Ausschusses für Rechtsfragen vom 3./4. Januar beschränkte sie sich weitgehend auf eine Zuschauerrolle: Ihre anwesenden Vertreter hielten sich bei den zentralen Fragen über das verstärkte justizpolitische Engagement der SED auffallend zurück und meldeten sich nur bei einem weitaus weniger wichtigen Thema zu Wort ${ }^{55}$.

Erst am 4. März 1948 zitierte die SMAD-Rechtsabteilung die DJV-Spitze und zwei Dolmetscher für den nächsten Tag zu einer Besprechung nach Karlshorst über „weitere Maßnahmen zur Demokratisierung der deutschen Justizorgane“. Neben Schiffer und Melsheimer mußten mit Benjamin, Winkelmann, Gentz und Hartwig die Leiter der Abteilungen II, III, IV und VI sowie die beiden kommunistischen Referenten der Abteilung V, Weiß und Nathan, nicht aber die sozialdemokratischen Leiter der Abteilungen I und V, Rosenthal-Pelldram und Karl Guski, teilnehmen. Bereits damit setzte die Rechtsabteilung ein Signal, daß die SPD-Genossen, die nach der Zwangsvereinigung nicht zur SED gewechselt waren, von nun an einen schweren Stand in der DJV haben würden ${ }^{56}$. Auf sowjetischer Seite waren nicht nur Karassjow und sein Stellvertreter Bukanow, sondern mit Jakupow, Dozenko, Jeroma und Nikolajew noch vier weitere Offiziere der Rechtsabteilung zugegen. Während Dozenko lediglich eine „durchgreifende Entnazifizierung der deutschen Gesetze", insbesondere des Gerichtsverfassungsgesetzes, der Strafprozeßordnung und einzelner Wirtschaftsgesetze anmahnte, legte Jakupow mit vernichtender Kritik die angeblichen Mängel des Justizwesens offen, die insbesondere durch ein verstärktes Engagement der DJV behoben werden sollten. Dabei führte er als erstes die Notwendigkeit an, die „reaktionäre Gesinnung [in der Justiz] vollständig auszumerzen", um dann im einzelnen auf die noch in Kraft befindlichen NS-Gesetze, auf die "Neue Justiz“, Auswahl, Ausbildung und Einsatz der Volksrichterschüler, die juristischen Fakultäten, die zu milde Rechtsprechung im Zusammenhang mit Befehl Nr. 201, die Verfolgung der Wirtschaftsdelikte sowie die mangelhafte Nutzung des Statuts durch die DJV gegenüber den Länderregierungen tadelnd einzugehen. Wenngleich nicht alle Mängel

52 Abteilung Justiz an Fechner/Ulbricht, 1. 12. 1947, BAB, DP1 VA Nr. 6596, Bl. 108.

53 Vgl. Naimark, Russians in Germany, S. 35. Für die Hilfe bei der Datierung dieser zitierten Äußerung danke ich Dr. Jan Foitzik.

$5+\mathrm{Vgl}$. Bordjugov, Rechtsabteilung, S. $64 \mathrm{f}$.

55 Deren Anwesenheit geht aus der Begrüßung Schäfermeyers hervor; die Äußerungen Jakupows bezogen sich auf das gerichtliche Vorgehen gegen Verstöße gegen die Ablieferungspflicht: siehe Stenographische Niederschrift über die 3. Tagung des Ausschusses für Rechtsfragen beim Zentralsekretariat der SED, 3./4. 1. 1948, SAPMO, DY 30 IV 2/1.01/70, Bl. 3, 35-37.

56 Vermerk über ein Telefonat Jakupows, 4. 3. 1948, BAB, DP1 VA Nr. 11, Bl. 129. Keines der SPDMitglieder in der DJV war nach der Gründung der SED aus seiner Partei ausgetreten: siehe Kap. A.I.4. 
von der DJV zu verantworten waren - Schiffer wies in seiner Entgegnung unter anderem darauf hin, daß das Statut der DJV zur Klärung des Verhältnisses zu den Länderregierungen nicht ausreiche -, war deutlich geworden, daß die justizpolitischen Ziele und Mittel der SMAD-Rechtsabteilung und der SED-Führung in einem hohen Maße übereinstimmten ${ }^{57}$.

Vor diesem Hintergrund ergab sich 1948 nachweisbar eine enge Kooperation zwischen SMAD-Rechtsabteilung und der SED-Führung in der Justizpolitik. Als Jakupow am 19. Mai 1948 die Abhaltung einer Konferenz in der DJV zum 11./ 12. Juni anordnete, betraute er den justizpolitischen Sprecher der SED mit dem Hauptreferat, „Aufgaben der weiteren Demokratisierung der Justiz“, das, wie alle anderen Vorträge auch, vorab in Karlshorst einzureichen war ${ }^{58}$. Fechner erhielt somit die Möglichkeit, seine Thesen vor einem knapp hundertköpfigen Publikum zu verbreiten, das sich aus zahlreichen prominenten Vertretern der Länderjustiz, der politischen Parteien, der DWK, der DVdl, der DJV und der SMAD-Rechtsabteilung zusammensetzte ${ }^{59}$. Die drei anderen Referenten der DJV, Melsheimer, Weiß und Hirschfeld, behandelten die Durchführung von Befehl Nr. 201 und wirtschaftsstrafrechtliche Fragen. Schiffer, der bereits im April mit seinem Vorschlag zur Reform des Gerichtsverfassungsgesetzes an dem Widerspruch der SED-Justizabteilung und der SMAD-Rechtsabteilung gescheitert waroo, kam auf dieser Tagung lediglich eine Statistenrolle zu; damit wurde unübersehbar, daß seine Zeit als aktiver, die Justizentwicklung maßgeblich beeinflussender DJV-Präsident zu Ende ging. Die SED-Justizabteilung sah damals indes noch nicht den Zeitpunkt gekommen, um ihn endgültig zu entfernen. Sie war vielmehr bestrebt, dessen „Opposition“ und „Mißtrauen“ im Hinblick auf die Konferenz vom Juni $\mathrm{zu}$ beseitigen, indem ihm gegenüber hervorgehoben wurde, daß die "Justizlinie der SED [...] vor allem in der Wiederherstellung und Schaffung aller Rechtsgarantien und dem Ausbau der Autorität der Staatsanwaltschaft und Gerichte" liege ${ }^{61}$.

Dementsprechend enthielten Fechners Ausführungen vom 11. Juni - im Unterschied zu seinen vorangegangenen Reden vor SED-Gremien - keine unverblümten Aussagen zum verstärkten Eingreifen der Partei in die Justiz; ohne die SED zu nennen, vertrat er indes deren Positionen zur Unabhängigkeit der Justiz, zum Verhältnis von Richter und Politik, zur Volksrichterfrage, zum Strafvollzug und zum Wirtschaftsstrafrecht ${ }^{62}$. Jakupow, der ebenfalls in die Debatte eingriff, argumentierte in eine ähnliche Richtung, als er sich unter anderem für „ein zielbewußteres Zuziehen der Vertreter des Volkes zur Rechtspflege" mittels der Volksrichter und gegen die Ansicht aussprach, „daß der Richter unpolitisch sein müsse“. Vor dem Schlußwort Schiffers wies Karassjow nochmals auf die Bedeutung von Fech-

57 Bericht über die Besprechung bei der Rechtsabteilung in Karlshorst am 5. 3. 1948, ebenda, Bl. 130135. Auch auf Landesebene versuchte die SMA „seit 1948 die Zügel in der Justizpolitik enger zu ziehen": vgl. Pohl, Justiz in Brandenburg (Manuskript), S. 96.

58 Aktenvermerk über den Besuch Jakupows in der DJV am 19. 5. 1948, in: Amos, Justizverwaltung, S. $234 \mathrm{f}$.

59 Siehe die Anwesenheitsliste in: Protokoll über die Juristenkonferenz der DJV vom 11./12. 6. 1948, BAB, DP1 VA Nr. 264, Bl. 1-4; vgl. Amos, Justizverwaltung, S. 64.

60 Siehe dazu Kap B.IX.3.

61 Abt. Justiz an Fechner, 27. 4. 1948, BAB, DP1 VA Nr. 6596, Bl. 12.

62 Das Referat Fechners ist gedruckt in: NJ 2 (1948), S. 121-126. 
ners Vortrag hin und mahnte zu mehr Kritik und Selbstkritik in der Justiz ${ }^{63}$. Nach dem Willen der SMAD-Rechtsabteilung schloß sich im Juli und August 1948 eine Serie ähnlicher Konferenzen mit der gleichen Tagesordnung in den einzelnen Ländern an, die von denselben Referenten bestritten wurden und fast alle Angehörigen der Justiz erreichten ${ }^{64}$. Den Abschluß bildeten jedes Mal fast durchweg einstimmig angenommene, weitgehend identische Resolutionen, die die justizpolitischen Ziele der SED bekräftigten ${ }^{65}$.

Damit war der Tagungsreigen immer noch nicht beendet, da nun überprüft werden mußte, ob die mit den Landesjustizkonferenzen gestellten Aufgaben auch in Angriff genommen wurden. Dabei ergriff nicht die DJV, sondern das SED-Zentralsekretariat die Initiative. Denn bereits am 18./19. August gingen von Fechner unterzeichnete Schreiben an die Landesvorsitzenden, in denen unter Bezugnahme auf die Landeskonferenzen die justizpolitischen Aufgaben der nächsten Zeit skizziert und eine zweite zentrale Justiztagung der DJV im Herbst angekündigt wurde, „auf der die Vertreter der Länder über das bisher Durchgeführte zu berichten haben"66. Erst am 20. September trat die DJV in derselben Angelegenheit an die Landesjustizministerien heran. Die Rundverfügung konkretisierte, in Anlehnung an Fechners Ausführungen vom Juni, die Aufgaben der Justizverwaltungen im Hinblick auf die Aus- und Weiterbildung des Justizpersonals, die Personalpolitik, die stärkere Beteiligung der Laien in der Justiz, die Zusammenarbeit der Justiz mit der Verwaltung, den Strafvollzug sowie die Durchführung von Befehl Nr. 201 und wirtschaftsstrafrechtliche Fragen ${ }^{67}$. Wenngleich ohne erkennbare Beteiligung der SMAD-Rechtsabteilung initiiert, war diese von der Tagung bereits unterrichtet, als die DJV ihr am 22. Oktober die Tagesordnung übermittelte. Neben der Berichterstattung der Ländervertreter waren Referate über „Zweijahresplan und Justiz" (Heinrich Rau), die Wirtschaftsstrafverordnung (Wolfgang Weiß), die Kontrollkommissionen (Fritz Lange) und die besonderen Aufgaben der Justiz bei der Unterstützung der Arbeiten der Kontrollkommissionen (Hildegard Heinze) vorgesehen ${ }^{68}$. Da die Rechtsabteilung die Referate zu den Kontrollkommissionen gestrichen sehen wollte - wahrscheinlich in dem Bestreben, deren

63 Die Redebeiträge Jakupows und Karassjows in: Protokoll über die Juristenkonferenz der DJV vom 11./12. 6. 1948, BAB, DP1 VA Nr. 264, Bl. 19-22, 27-29.

$64 \mathrm{Vgl}$. die mündliche Anordnung Karassjows am 12. 6. 1948, ebenda, Bl. 29, und den Aktenvermerk über den Besuch Jakupows in der DJV am 19. 5. 1948, in: Amos, Justizverwaltung, S. 235. Die sowjetische Anordnung wurde umgesetzt in einer Rundverfügung der DJV vom 18.6. 1948, BAB, DP1 VA Nr. 6899. Für die Verbreitung der Ergebnisse der Konferenz sorgte auch der Abdruck der Referate und eine Zusammenfassung der Diskussion in der Neuen Justiz: siehe Juristenkonferenzen.

65 Siehe Mitteilungen der Abteilungen Justiz des Zentralsekretariats der SED, August 1948, SAPMO, DY 30 IV 2/13/407. Die als erste verabschiedete Weimarer Resolution wurde von den anderen Länderkonferenzen nur um einen Schlußteil ergänzt. Dies geht aus dem Druck in: NJ 2 (1948), S. 139 f., nicht eindeutig hervor.

66 An die Vorsitzenden der Landesleitung der SED Sachsen, 18. 8. 1948, BAB, DP1 VA Nr. 261, Bl. 2-6, hier 5. An die Vorsitzenden der Landesleitung der SED Mecklenburg, 19. 8. 1948, BAB, DP1 VA Nr. 20, Bl. 26-30, hier 29. Die Schreiben sind textidentisch.

67 Chef der DJV an Landesregierungen/Justizministerium, 20. 9. 1948, BAB, DP1 VA Nr. 6335; Teildruck in: Wentker, Volksrichter, Dok. 39, S. 224-226.

68 Chef der DJV an SMAD-Rechtsabteilung, 22. 10. 1948, BAB, DP1 VA Nr. 6589. Daß diesem Schreiben bereits ein Diskussionsprozeß vorangegangen war, zeigt die Bezugnahme auf "mündliche und telefonische Rücksprache mit Herrn Nikolajew“. 
keineswegs rechtsstaatskonformes Wirken möglichst geheim zu halten -, referierte statt dessen Nathan über das Thema „Rechtsschutz für die Errungenschaften des demokratischen Aufbaus (zivilrechtliche Fragen)" 69 .

Der 74-köpfige Teilnehmerkreis der zweiten Justizkonferenz vom 25./26.November war gegenüber der Juni-Veranstaltung um den Vorsitzenden der Zentralen Kontrollkommission, Fritz Lange, und um Vertreter des bei der DWK angesiedelten Ausschusses zum Schutze des Volkseigentums erweitert worden: zwei im Mai 1948 neu gegründeten Einrichtungen, die vor allem in Wirtschaftsstrafsachen auf Kosten der Justiz erhebliche Kompetenzen erhalten sollten. Auch die SMADRechtsabteilung war präsent. In seiner einzige Wortmeldung erklärte Jakupow ganz allgemein die „Demokratisierung der Justiz“ zur Hauptaufgabe, trat sonst in der Diskussion aber nicht weiter hervor. Bemerkenswert war vielmehr, mit welcher Offenheit Fechner verkündete, daß man „nicht mehr vom subjektiven Recht des einzelnen ausgehen" könne, sondern daß "die vordringliche und erstrangige Aufgabe der Justiz" im Schutz des Wirtschaftsplans und des Volkseigentums bestehe. Damit propagierte er - nicht mehr als Parteipolitiker, sondern als DJV-Präsident - vor den Landesjustizministern das instrumentelle Justizverständnis der SED. Die Konferenz war nur noch bedingt ein Forum des freien Austauschs, jedenfalls nicht in ihrem ersten Teil, in dem die Justizminister über den Stand der "Demokratisierung der Justiz" Bericht erstatten und sich kritische Hinweise Melsheimers und Benjamins gefallen lassen mußten. In die gleiche Richtung wie deren Beanstandungen gingen schließlich die Entschließungen, die neben Benjamin und Weiß auch von je einem Ländervertreter ausgearbeitet worden waren, aber dennoch eindeutig die Handschrift der DJV-Spitze trugen ${ }^{70}$. Indem in den Resolutionen zur Personalpolitik, zur Aus- und Weiterbildung, zum Themenkomplex Justiz und Öffentlichkeit, zu den Verfahren nach Befehl Nr. 201 sowie zu den einzelnen Referaten - insbesondere zum Wirtschaftsstrafrecht - weitere Aufgaben gestellt wurden, sollte die seit Jahresbeginn angestoßene Mobilisierung in der Justiz der SBZ in Gang gehalten werden, um, wie es in dem offiziösen Bericht in der Neuen Justiz hieß, "dadurch den Prozeß der durchgreifenden Demokratisierung der Justiz in der sowjetischen Besatzungszone weiter voranzutreiben und zu vollenden" 71 .

Bei der programmatischen Wende im Justizwesen, die 1947 vorbereitet und 1948 vollzogen wurde, handelte es sich um einen vornehmlich von der SED getragenen Prozeß, die damit zum ersten Mal seit 1946 auch auf diesem Terrain als richtungweisende Kraft auftrat. Dabei stand ihr justizpolitisches Engagement in engem Zusammenhang mit ihrem im Vorfeld der ersten Parteikonferenz im Januar 1949 immer stärker betonten gesamtgesellschaftlichen Führungsanspruch. Wenngleich die SED-Führung dabei die treibende Kraft war, wurde sie in ihrem Vorhaben, die SBZ zur „Volksdemokratie“ umzuwandeln, von der Besatzungsmacht weitgehend - wenn auch nicht bis zur letzten Konsequenz - unterstützt ${ }^{72}$. Die

69 Vermerk über Telefonat mit Jakupow, 2. 11. 1948, ebenda; Vermerk, 3. 11. 1948, ebenda.

70 Protokoll über die 2. Juristenkonferenz in Berlin am 25./26.11. 1948, BAB, DP1 VA Nr. 839, Bl. 2-19, insbesondere Bl. 5 (Zitat Fechner), 12f. (Melsheimer), 17 (Benjamin), 15f. (Jakupow).

71 Zweite Juristenkonferenz, S. 265; die Resolutionen ebenda, S. $266 \mathrm{f}$.

72 Vgl. Staritz, SED, Stalin und der Aufbau des Sozialismus. 
SMAD-Rechtsabteilung trat gegenüber der SED zwar als Motor der Veränderungen im Justizwesen um die Jahreswende 1947/48 zurück, handelte jedoch ab März 1948 in Übereinstimmung und Kooperation mit der Führung der Einheitspartei. Dies bildete die unerläßliche Voraussetzung für die intensivierte, nunmehr eindeutig erkennbare Transformation des Justizwesens in der SBZ. 